\title{
Research On Enterprise Service Marketing of UFIDA Software
}

\author{
Qian Bai \\ School of Management, Yulin University, China,719000 \\ 9393219363@qq.com
}

Keyword: UFIDA ; ERP ; Service mrketing ; Strategy

\begin{abstract}
The service marketing based on the proposed new UFIDA marketing model, and construct the ERP marketing system, make the research on service marketing of UFIDA aspects. Through analyzing the current situation of service marketing strategy of UFIDA software company at present, find its success, according to the research on this theory and practice analysis, pointed out that the UFIDA software the success of the company for other products management software industry. It not only has certain practical significance on UFIDA company's future development, but also to bring some experience of other enterprises for China's software industry.
\end{abstract}

\section{Service Marketing Status of UFIDA}

Product Strategy Status. FIDA is Asia's largest local ERP software supplier, Chinese is the largest management software, ERP software, financial software provider. In China ERP software market, the largest market share of UFIDA software, the rich product line, the most successful applications, the industry's most extensive coverage, the largest service network, the delivery capacity of the strongest UFIDA software company currently. Its main products include NC, U8, U9 and T series of products, but because of the particularity of the software product, need to have higher professional technical support services, in order to be more accurate and perfect to meet the needs of customers, in the sale of any products, UF will provide consulting, training, implementation, customer development, operation and maintenance is a supporting service.

Price Strategy Status. UFIDA is a high-end management software (e-commerce) later, it uses the cost oriented pricing pricing, and the ERP software industry. Most enterprises UF price system is divided into two parts, one part is the basic price of the software, the other part is added in the software service price. The software price is selected according to the customer and offer different different modules. Whether NC, U8, U9 and T series of products, their prices are divided into three categories: basic module module, class module and financial supply chain module. The basic modules including general ledger, fixed assets, business report is basic the general module required by customers, price between 1-8 million; the financial module includes the consolidated financial statements, comprehensive budget, reimbursement, funds management and so on; supply chain module including purchasing, sales, inventory, contract and so on, the two belong to the extended module, enterprises can according to their actual needs to choose the price. Relatively high in the 20 thousand to 400 thousand range. Service price is calculated separately, according to the business needs of different levels and different consultant fees, consultant based on tenure and professional level is divided into expert, consultant, senior consultant, intermediate consultant, senior consultant and the service fee specifically in the table below: expert service fee per person per day from 6000 to 8000 yuan per person per day, consulting service fee: $4500-5000$, senior consultant service fee: 3500-4000 per person per day, the intermediate advisory service fee: $2500-3000$ per person per day, Junior Consultant Service Fee: 1500-2000 yuan per person per day.

Channel Strategy Status. UFIDA existing distribution channel mode is a hybrid, both indirect distribution channels have direct distribution channels. Indirect distribution channels, the agent in the area where the UF authorized agents, i.e. different series of products to choose different sales agents, the authorized agents of UFIDA software sales and services in the designated area. Direct distribution, UF headquarters under the large area, large area under the local branch. The headquarters is responsible for the management of each region; large area is responsible for the promotion and management of UFIDA software sales and service business in the area within the 
jurisdiction of branch management, scope, and to undertake ERP projects, large and medium sized enterprises such as: northeast region, central region; branch is divided according to the provinces, to undertake projects within the area under the jurisdiction of ERP, UFIDA is currently in the country except Tibet autonomous region are divided Company, such as: UFIDA Beijing branch, UFIDA Shaanxi branch and so on.

Promotion Strategy Status. IDA software marketing strategy is mainly has two aspects: one is the promotion, promotional activities, promotional activities are solved by market publicity successful application cases and industry solutions, mainly in order to establish a good brand image, at the same time to let customers have a certain understanding of the corresponding products, and products of interest arouse the customer demand. The other one is to price promotions, through discount, plus products and services such as marketing. ERP software industry promotion strategy used by most enterprises with similar products. The main price discount price promotions, buy gifts to buy the product service, free implementation, purchase products to deliver training the purchase, delivery service and so on. For example, in 2011 began to upgrade the TTU upgrade of service marketing strategy, customers can enjoy a free upgrade service.

Service Personnel Strategy Status. UF service in the domestic industry has a good reputation. The first is the implementation of the consultant UF management experts, according to the actual situation of the enterprise, the design of the new management mode and organization mode for the enterprise and to optimize the design of the business process. They are distinguished by expert software should be able to combine with their business solutions and applications do the full combination. Besides they also have excellent project management skills and ability of communication and coordination to mobilize internal and external resources to successfully promote the project process. Because the UF has the recruitment and training mechanism in a set of the UF has a group of excellent service personnel, so far, 500 UF UF consultant certification in the national scope for customers in different regions to provide implementation services, including 200 project manager and senior consultant, 300 name Shi Gu Asked.

\section{The Success of UFIDA Software Service Marketing}

Rich Products and Services. Currently, UF has formed a rich software and services product line, can fully meet the needs of customers. The software such as the most prominent representative of the difference between the UF two products U8 and NC.U8 and NC are the most essential U8 the main customer groups for the single organization of enterprises, a company a a set of books, does not support the merger, while NC is the main customer groups for multi enterprise group organization, the group used a set of books, support the merger. From the functional perspective, the function of NC is greater than U8, the world's top 500 enterprises in the use of all $80 \%$ companies are NC products, its solution to include two parts of the group control and industry management, maximize the comprehensive application of production and marketing integration, but the flexibility from the point of view, U8 is more flexible, and the U8 price also is much lower than the NC, almost to culvert Cover all small businesses business, is very suitable for small and medium enterprises. Such targeted design can fully meet the needs of customers, greatly improving the UF market share. The service according to the customer's buying process, provide consulting, training, implementation, customer development, operation and maintenance is a supporting service also, can fully meet the needs of customers.

Excellent Service Personnel. UF's success with its professional services are inseparable, in the service personnel selection, training UFIDA attaches great importance to. UF dedicated to establish a system of personnel training of ERP, the advanced information technology, advanced management concept and enterprise management are combined, we focus on staff in all aspects of literacy in the recruitment, another each stage in the enterprise information management, providing a full range of different level personnel, multi level training services.

Standardized Service Process. The successful implementation of Yongyou cannot do without the guidance of the theory of scientific methods, the implementation method of close to the UF UF software, the provisions of the implementation of the project team the responsibilities and rights of 
both parties, the implementation of the action specification, tools, project management norms related to the implementation of a full range of content flow. Standard pre-sales consulting, shortening the project cycle before. The consultation stage according to the needs of customers, by industry experts to provide reasonable proposals and solutions to avoid the demand, most likely to be encountered in the process of project implementation difficulties caused the project tardiness situation, to maximize the protection of the system implementation success rate of implementation of the programme. The most favorable sale, the project cost control. Methods the UF with scientific theory, implementation tools, implementing template improvement, implementation consultant 300 thousand customer experience and high quality, can fully meet customer The business process and the actual demand, issued by the most preferential implementation plan for the customer, until after the customer satisfaction can be confirmed by the implementation of the system, this process is effectively avoided due to the demands of customers change in the process of implementation of project cost control, project cost control on the maximum extent, and maximize enterprise profit. Strong customer service maintenance, customer service to ensure the quality of operations. Based on years of experience in enterprise application of UF, the enterprise's industry characteristics and its own characteristics, which are designed to improve the management level of the enterprise information technology business consulting services, and help enterprises play the maximum effectiveness of ERP system to achieve continuous business improvement. UF provide information operations consulting services include: evaluation of operation the special condition of the system, business improvement, system expansion plan, when the system is running after a period of time, the user's requirements should be UF Consultant first operation of the existing system to make analysis and evaluation. According to the different results, UF offers different improvement programs. The existing system can not meet the needs of business improvement, provide system expansion scheme, guide enterprises to carry out the improvement of the system, upgrading, integration and help enterprises to further improve the construction of information system for the lack of effective application. The system does not achieve the optimization effect of UF to help companies develop business process optimization scheme, a clear corporate sector, the role of post establishment of quality management system and performance appraisal system, make the enterprise business management process according to the new smooth operation.

\section{The Inspiration Of Service Marketing for ERP Software Enterprises in China}

Customer Centered to Meet Customer Needs. he design of management software products should follow the "customer centered" attitude, customer requirements in accordance with the type of business, service type, firm size and other different dimensions are classified according to different categories of enterprises to go to belong to the ERP of their software, and should take into account the complexity and diversity of business process and consider a variety of special circumstances, combined with the previous customer experience, as far as possible to improve the degree of flexibility to adapt to a variety of products, customer demand. It can be said that the future of the ERP software industry should gradually to the custom development direction

In the field of management software, the user needs is not only a single product, but also need a series of professional, comprehensive service and support, should always regard customer as the center, business users in the process of purchasing management software products, related to the pre-sale, sale, customer service continued to plan, according to this purchase. Can be combined with training, consulting, implementation, customization, operation and maintenance, convenient service hotline support, version upgrades, network services, information services, customer service conference, product design and service in a cooperative alliance, to bring a full range of products to the customer experience. As to the customer as the center, to meet customer needs.

Cultivate High Quality Service Personnel to Enhance Customer Loyalty. F software attaches great importance to the training of service personnel, according to the different types of service personnel to develop different training content and flexible training program is not only a software product operation on personnel training, also attaches great importance to employee occupation 
accomplishment, training service providers, establish employee service consciousness, regulate employee service process the UF brand awareness and customer loyalty in the industry reputation.

Because of the special management software industry needs to have the professional skills of service personnel, so the service personnel in all aspects of literacy has very high requirements, the quality of products by service personnel and professional services to support, therefore timely to attract professional talents to enrich the marketing team is very important for the recruitment of marketing personnel. Not only need to constantly expand their professional knowledge, understand the product features, characteristics, also need to have good occupation accomplishment, including occupation morality, occupation, occupation and occupation style consciousness, so as to constantly improve its service level and management consciousness, so as to give advice and suggestions of enterprise marketing, this can be a very good upgrade customer loyalty and satisfaction. Now the business performance in the purchase of the product not only depends on the quality of the products and the good and bad in itself, and enterprises pay more attention to the good attitude Good. So consumers will pay more attention to consider whether they will meet in the purchase of the product, the enterprise service consciousness can meet the current consumer demand is a severe test. First, enterprises should realize that the real in service marketing, service is the essence of the goods is highly prized, and the product is service accessories. Only in this way can the enterprise is on service marketing of a certain level of understanding, and continuously strive to improve enterprise staff service consciousness. Secondly, the service is not only limited and enterprise service personnel, every employee of the enterprise will become the company's marketing representative ", can be in processing all kinds of problems of consumers, and then display the enterprise culture and the strength in front of consumers. For example, the implementation of technical personnel and skilled operation maintenance personnel can well cultivate customer loyalty, good The old customer management, prompting the old customers long-term purchase of the company's operation and maintenance services, and even buy module

Building a Perfect Service Process. roject implementation methodology is a detailed framework to guide the whole UF project implementation, it has various stages of the project implementation activities organized project organization, defines a set of document system best practice base, describing the work procedures in detail, and the task of each stage of the project milestone, standardized project management of the implementation process, great to improve work efficiency, reduce the cost of the project.

In the pre-sale consultation stage, and its communication with customers should understand the customer demand, the real business scene, issued by the project proposal and the task of the book and signed with customers. After all, ERP such a long time span of the project, involving many departments, and brain hardware participation, there may be risks, so be sure to agree with customers early in the project, in order to effectively avoid project risk. The implementation in the sale stage, shall be issued by the industry expert consultants to improve the implementation of the program, in a test environment as far as possible the testing scheme more rational and applicable scheme can ensure the smooth operation, and can help customers improve work efficiency, standardize the business process. In the customer service operation support system should be established in the internal, at any time on-line monitoring problems and timely solve, do training for customers with assessment of certain factors, do Effective knowledge transfer, avoid repeated training work, only in this way can ensure the acceptance of the late project can be carried out smoothly as scheduled

This perfect service process makes the UF one step up today, is of great significance for the ERP software industry in our country, the same industry ERP software enterprises should rely on many years of experience in enterprise application, according to the characteristics of the enterprise to develop a set of their own, for their service system.

\section{References}

[1] Li Xuemei. Discussion on the micro-blog marketing [J]. modern economic information, 2014, (4): 349. 
[2] u Ning. Internal marketing -- the basis of competitive advantage of management software enterprises [J]. science and technology information development and economics, 2015 (19): 47-49.

[3] ang Yi. Some thoughts on developing service industry [J]. new Long March, 2016 (6): 8-9.

[4] hu Yandan, Jing Ming. Entertainment V communication behavior and the influence of [J]. news fans, 2015, (6): 58-62.

[5] iang Haihong. Analysis of competitive advantage of management software enterprises based on customer value [J]. business research 2015 (03): 132-133.

[6] F Limited by Share Ltd document small business division. Enterprise information software technology development trend analysis report [J]. business research 2015 (1): 7-9. 